\title{
THE CONCORDANCE DIFFEOMORPHISM GROUP OF REAL PROJECTIVE SPACE
}

\author{
BY \\ ROBERT WELLS
}

\begin{abstract}
Let $P_{r}$ be $r$-dimensional real projective space with $r$ odd, and let $\pi_{0}$ Diff $^{+}$: $P_{r}$ be the group of orientation preserving diffeomorphisms $P_{r} \rightarrow P_{r}$ factored by the normal subgroup of those concordant (= pseudoisotopic) to the identity. The main theorem of this paper is that for $r=11 \bmod 16$ the group $\pi_{0} D_{\text {iff }}^{+}: P_{r}$ is isomorphic to the homotopy group $\pi_{r+1+k}\left(P_{\infty} / P_{k-1}\right)$, where $k=d 2^{L}-r-1$ with $L \geq \varphi((r+1) / 2)$ and $d 2^{L} \geq r+1$. The function $\varphi$ is defined by $\varphi(l)=\{i \mid 0<i \leq l, i=0,1,2,4 \bmod (8)\}$. The theorem is proved by introducing a cobordism version of the mapping torus construction; this mapping torus construction is a homomorphism $t: \pi_{0} \operatorname{Diff}^{+}: P_{r} \rightarrow \Omega_{r+1}(v)$ for $r=11$ mod 16 and $\Omega_{r+1}(v)$ a suitable Lashof cobordism group. It is shown that $t$ is an isomorphism onto the torsion subgroup $\Omega_{r+1}(v)$, and that this subgroup is isomorphic to $\pi_{r+1+k}\left(P_{\infty} / P_{k-1}\right)$ as above. Then one reads off from Mahowald's tables of $\pi_{n+m}\left(P_{\infty} / P_{m-1}\right)$ that $\pi_{0}$ Diff $^{+}: P_{11}=Z_{2}$ and $\pi_{0}$ Diff $^{+}: P_{27}=6 Z_{2}$.
\end{abstract}

Let $P_{r}$ be $r$-dimensional real projective space, and let $\pi_{0}$ Diff $^{+}: P_{r}$ be the group of orientation preserving diffeomorphisms $P_{r} \rightarrow P_{r}$ factored by the normal subgroup of those concordant (= pseudoisotopic) to the identity. The object of this paper is to determine an isomorphism from the group $\pi_{0}$ Diff: $P_{r}$ for $r \equiv 11$ mod 16 to the torsion subgroup of a certain Lashof cobordism group. To define the cobordism group, let the fibration $v: P[l] \rightarrow B S O$ be such that $P_{\infty} \rightarrow P[l]$ $\stackrel{\hookrightarrow}{\longrightarrow} B S O$ is the lth stage in the Moore-Postnikov decomposition of the map $P_{\infty} \rightarrow B S O$ classifying an orientation of $k y_{\infty}$, where $y_{r} \rightarrow P_{r}$ is the canonical line bundle, $l=(r+1) / 2$ and $k=d 2-r-1$ for $L \geq \varphi(l)$ and $d 2 \geq r+1$; recall that $\varphi(l)=\#\{i \mid 0<i \leq l, i \equiv 0,1,2,4 \bmod 8\}$. Let $\Omega_{r+1}(v)$ be the $(r+1)$ st

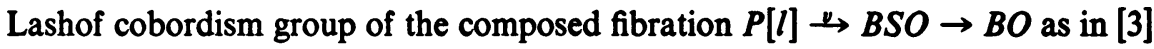
or [6]. Finally, let $\zeta: P_{r} \rightarrow P_{r}$ be the diffeomorphism defined by the matrix

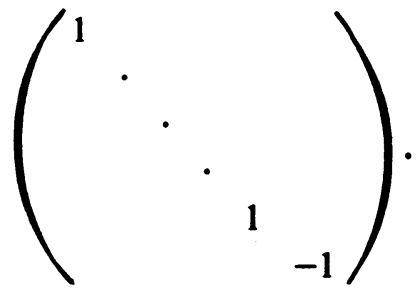

Then the theorem is the following.

Received by the editors September 7, 1972 and, in revised form, January 15, 1973.

AMS (MOS) subject classifications (1970). Primary 57D50, 57D90; Secondary 55El0.

Key words and phrases. Diffeomorphism, concordance, pseudoisotopy, Lashof cobordism groups, Moore-Postnikov decomposition, mapping torus, surgery, Wall groups.

Copyright $\odot$ 1974, American Mathematical Society 
Theorem 1. There is an isomorphism $t: \pi_{0}$ Diff $^{+}: P_{r} \rightarrow$ Tors $\Omega_{r+1}(v)$ for $r \equiv 11$ $\bmod 16$, and $t(\zeta \circ \varphi \circ \zeta)=-t(\varphi)$.

Corollary 1. For $r \equiv 11 \bmod 16, \pi_{0}$ Diff $^{+}: P_{r}$ is a finite abelian group.

Let $\pi_{0}$ Diff: $P_{r}$ be the groups of diffeomorphisms $P_{r} \rightarrow P_{r}$ factored by the normal subgroup of those concordant to the identity. Define a homomorphism $s: \pi_{0}$ Diff: $P_{r} \rightarrow Z_{2}$ by $s(\varphi)=1$ if $\varphi$ is orientation reversing and $s(\varphi)=0$ if $\varphi$ is orientation preserving. Then the sequence

$$
1 \rightarrow \pi_{0} \text { Diff }^{+}: P_{r} \rightarrow \pi_{0} \text { Diff: } P_{r} \stackrel{s}{\rightleftarrows} Z_{2} \rightarrow 0
$$

is exact, split as shown. The splitting is defined by $1 \rightarrow \zeta$. Thus $\pi_{0}$ Diff $^{+}: P_{r}$ with $Z_{2}$, the action of $Z_{2}$ on $\pi_{0}$ Diff $^{+}: P_{r}$ being defined by $0 \cdot \varphi=\varphi$ and $1 \cdot \varphi$ $=\zeta \circ \varphi \circ \zeta$. In the case $r \equiv 11 \mathrm{mod} 16$, we obtain the following corollary of Theorem 1.

Corollary 2. If $r \equiv 11 \bmod 16$, then $\pi_{0}$ Diff: $P_{r}$ is isomorphic to the semidirect product of Tors $\Omega_{r+1}(v)$ with $Z_{2}$, the action of $Z_{2}$ on Tors $\Omega_{r+1}(v)$ being defined by $0 \cdot x=x$ and $1 \cdot x=-x$.

The commutative diagram of fibrations

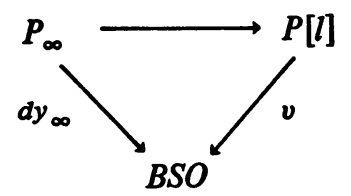

produces a homomorphism from the $k y_{\infty}$-cobordism groups to the $v$-cobordism groups. By the Thom theorem, the $k y_{\infty}$-cobordism groups are the stable homotopy groups of the truncated projective space. Thus we have a homomorphism $\pi_{r+1}^{s}\left(P_{\infty} / P_{k-1}\right) \rightarrow \Omega_{r+1}(v)$. Let $\gamma \rightarrow P_{l}$ be the $(l+1)$-plane bundle over $P_{l}$ stably equivalent to $(l-1) y_{l}$, and let $T(\gamma)$ be its Thom space. Let $j:(K O)^{\sim}(T(\gamma))$ $\rightarrow \pi_{r+1+k}^{s}\left(P_{\infty} / P_{k-1}\right)$ be the $j$-homomorphism defined in [8].

Lemma. The following sequence is exact:

$$
(K O)^{\sim}(T(\gamma)) \stackrel{\leftrightarrow}{\rightarrow} \pi_{r+1+k}^{s}\left(P_{\infty} / P_{k-1}^{*}\right) \rightarrow \operatorname{Tors}\left(\Omega_{r+1}(v)\right) \rightarrow 0 .
$$

Proof. According to [8], there is an isomorphism $I_{r+1}(k) \rightarrow \wedge_{r+1}(k)$, where $\Lambda_{r+1}(k)$ is the cokernel of $j$, and $I_{r+1}(k)$ is the group of free involutions of type $k$ on homotopy $S^{(r+1) / 2} \times S^{(r+1) / 2}$ s; for details see [5], [8]. According to $H$. Schneider [5] there is an isomorphism $I_{r+1}(k) \cong \operatorname{Tors}\left(\Omega_{r+1}(v)\right)$. Moreover, it is straightforward to check that the diagram

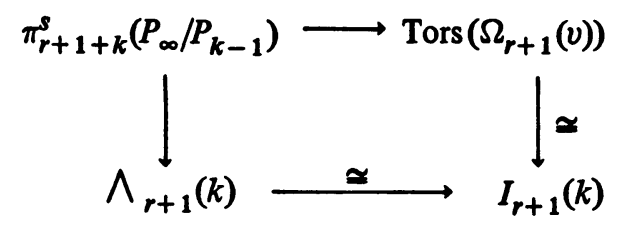


commutes, where the bottom map is the inverse of the inverse of the isomorphism $I_{r+1}(k) \rightarrow \bigwedge_{r+1}(k)$. The lemma follows.

From Theorem 1 and the lemma above, we obtain immediately the following theorem.

Theorem 2. Let $r \equiv 11 \bmod 16, r+1=2 l, k=d 2^{L}-r-1$ where $L \geq \varphi(l)$ and $d 2^{L} \geq r+1$. Then:

(1) $\pi_{0} \mathrm{Diff}^{+}: P_{r} \cong \pi_{r+1+k}\left(P_{\infty} / P_{k-1}\right)$.

(2) $\pi_{0}$ Diff: $P_{r}$ is isomorphic to the semidirect product of $\pi_{r+1+k}\left(P_{\infty} / P_{k-1}\right)$ with $Z_{2}$, the action of $Z_{2}$ on $\pi_{r+1+k}\left(P_{\infty} / P_{k-1}\right)$ being defined by $0 \cdot X=X$ and $1 \cdot X=X$.

Proof. For $\gamma$ as above, $(K O) \sim(T(\gamma))=0$.

Corollary.

$$
\begin{aligned}
\pi_{0} \text { Diff }^{+}: P_{11}=Z_{2}, & \pi_{0} \text { Diff }^{+}: P_{27}=6 Z_{2}, \\
\pi_{0} \text { Diff: } P_{11}=2 Z_{2}, & \pi_{0} \text { Diff: } P_{27}=7 Z_{2} .
\end{aligned}
$$

Proof. Read $\pi_{r+1+k}\left(P_{\infty} / P_{k-1}\right)$ off Mahowald's tables [4]. There is no odd torsion in $\pi_{r+1+k}\left(P_{\infty} / P_{k-1}\right)$ for $r=11,27$ by results of J. P. May or J. Cohen [13], [14].

Theorem 2. Let $r \equiv 11 \bmod 16 ; r+1=2 l$; and $k=d 2^{L}-r-1$ where $L \geq \varphi(l)$ and $d 2^{L} \geq r+1$. Then $\pi_{r+1+k}^{s}\left(P_{\infty} / P_{k-1}\right)$ has a direct summand $Z_{2}$.

Proof. For $\gamma$ as above, $K O(T(\gamma))=0$.

Remark. The following weaker theorem may be easily obtained by means of well-known methods:

Theorem 2. Let $r \equiv 11 \bmod 16, r+1=2 l$ and $k=d 2^{L}-r-1$ where $L \geq \varphi(2 l-1)$ and $d 2^{L} \geq r+1$. Then there is a nontrivial element of order two in $\pi_{r+1+k}^{s}\left(P_{\infty} / P_{k-1}\right)$.

Proof. Since $L=\varphi(2 l-1)=\varphi(r)$ there is a commutative diagram

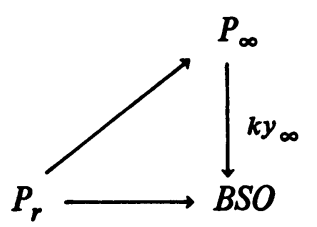

with the horizontal map a classifying map for the (oriented) normal bundle of $P_{r}$. Then $P_{r} \rightarrow P_{\infty}$ represents an element of the $r$ th cobordism group of $k y_{\infty}$; by the Thom theorem that is an element $\alpha \in \pi_{r+k}^{s}\left(P_{\infty} / P_{k-1}\right)$. Let $y \in \pi_{1}^{s}$ be the nonzero element. Then $y \cdot \alpha \in \pi_{r+1+k}^{s}\left(P_{\infty} / P_{k-1}\right)$. The cobordism class of $P_{r} \rightarrow P_{\infty}$ has a nonzero characteristic number.

Notation. If $M$ is a submanifold of $N$, then $v(M: N)$ is the normal bundle of $M$ in $N$. If $\xi \rightarrow X$ is a vector bundle, then $E(\xi) \rightarrow X$ is the associated disk bundle 
and $S(\xi) \rightarrow X$ is the associated sphere bundle. $R_{+}^{n}=\left\{\left(x_{1}, \ldots, x_{n}\right) \mid x_{i} \in R, x_{n}\right.$ $\geq 0\}$.

1. Preliminaries. For $M$ a smooth manifold and $X \subset M$ let $\operatorname{Diff}(M, X)$ be the group of diffeomorphisms of $M$ fixed on $X$; let $C(M, X)$ be the normal subgroup of those concordant mod $X$ to the identity. Following Antonelli-Burghelea-Kahn [1], we write $\pi_{0}$ Diff: $(M, X)$ for the quotient group $\operatorname{Diff}(M, X) / C(M, X)$, and $\pi_{0}$ Diff $^{+}:(M, X)$ for the subgroup represented by orientation-preserving diffeomorphisms. If $X=\varnothing$ we write $\pi_{0}$ Diff: $M$. We extend this notation to embeddings. Let $V$ be a smooth manifold and let $j: X \rightarrow V$ be a map that extends to an embedding in $V$ of $M$. Write $\xi(M, j: V)$ for the set of embeddings of $M$ in $V$ extending $j$. If $X=\varnothing$ we write $\xi(M: V)$. Let $\pi_{0} \xi:(M, j: V)$ be the quotient of $\xi(M, j: V)$ by the equivalence relation of concordance $\bmod j$. Fix $\varphi: M \rightarrow V$ an embedding extending $j$-it will be the basepoint in the definition of $\pi_{1} \xi$ : $(M, j: V)_{\varphi}\left(\varphi\right.$ will be omitted if the basepoint is clear). Let $j_{\varphi}: M \times[0, \varepsilon) \cup X \times I$ $\cup M \times(1-\varepsilon, 1] \rightarrow V \times I$ be defined by $(x, t) \rightarrow(\varphi(x), t)$. Then we set $\pi_{1} \xi$ : $(M, j: V)=\pi_{0} \xi:\left(M \times I, j_{\varphi}: V \times I\right)$. We have the obvious inclusion and restriction maps

$$
\operatorname{Diff}\left(P_{2 l-1}, P_{l-2}\right) \rightarrow \operatorname{Diff}\left(P_{2 l-1}\right) \Rightarrow \xi\left(P_{l-2}: P_{2 l-1}\right)
$$

and

$$
\operatorname{Diff}\left(P_{2 l-1}, N\right) \rightarrow \operatorname{Diff}\left(P_{2 l-1}, P_{l-2}\right) \rightarrow \xi\left(N, L: P_{2 l-1}\right),
$$

where $L: P_{l-2} \rightarrow P_{2 l-1}$ is the inclusion, which give rise to maps

$$
\pi_{0} \text { Diff: }\left(P_{2 l-1}, P_{l-2}\right) \rightarrow \pi_{0} \text { Diff: } P_{2 l-1} \stackrel{a_{0}}{\rightarrow} \pi_{0} \xi:\left(P_{l-2}: P_{2 l-1}\right)
$$

and

$$
\pi_{0} \text { Diff: }\left(P_{2 l-1}, N\right) \rightarrow \pi_{0} \text { Diff: }\left(P_{2 l-1}, P_{l-2}\right) \stackrel{\partial_{0}}{\rightarrow} \pi_{0} \xi:\left(N, L: P_{2 l-1}\right) \text {. }
$$

The left map in either case is a homomorphism. The middle group in each case acts on the right term, and the right map in each case is equivariant. Finally, it is easy to see that each of the sequences above is exact. We wish to extend these sequences by one term; to do so we define maps

$$
\pi_{1} \xi:\left(P_{l-2}: P_{2 l-1}\right)_{L} \stackrel{a_{1}}{\rightarrow} \pi_{0} \text { Diff: }\left(P_{2 l-1}, P_{l-2}\right)
$$

and

$$
\pi_{1} \xi:\left(N, L: P_{2 l-1}\right) \stackrel{\theta_{1}}{\rightarrow} \pi_{0} \text { Diff: }\left(P_{2 l-1}, N\right)
$$

as follows.

Construction of $\partial_{1}$. Suppose $x \in \pi_{1} \xi:\left(P_{l-2}: P_{2 l-1}\right)_{L}$ is represented by the embedding $\chi: P_{t-2} \times I \rightarrow P_{2 l-1} \times I$ such that $\chi(y, t)=(y, t)$ for $t$ near 0 and $t$ near 1 . Clearly there is an extension of $\chi$ to a diffeomorphism $\bar{\chi}: P_{2 l-1} \times I \rightarrow P_{2 l-1} \times I$ such that $\bar{\chi}(y, t)=(y, t)$ for $t$ near 0 and $\bar{\chi}(y, t)=(\varphi(y), t)$ for $t$ near 1 where $\varphi$ is a diffeomorphism $P_{2 l-1} \rightarrow P_{2 l-1}$ fixed on $P_{l-2}$. We define $\partial_{1} x$ to be element of 
$\pi_{0}$ Diff: $\left(P_{2 l-1}, P_{l-2}\right)$ represented by $\varphi$. If $\overline{\chi^{\prime}}$ is another extension as above, leading to a diffeomorphism $\varphi^{\prime}$, then $\bar{\chi}^{-1} \circ \bar{\chi}^{\prime} \mid P_{1-2} \times I=$ id so that $\varphi^{-1} \circ \varphi^{\prime}$ and id represent the same class in $\pi_{0}$ Diff: $\left(P_{2 l-1}, P_{l-2}\right)$; that is $\varphi$ and $\varphi^{\prime}$ represent the same class, and $\partial_{1} x$ is well defined to be the class of $\varphi$.

$\partial_{1}$ is a homomorphism. Suppose $x, x^{\prime} \in \pi_{1} \xi:\left(P_{l-2}: P_{2 l-1}\right)$. We may choose representatives $\chi, \chi^{\prime}: P_{l-2} \times I \rightarrow P_{2 l-1} \times I$ such that each is the identity near $P_{t-2} \times 0$ and $P_{t-2} \times 1$ and such that $\chi(y, t)=(y, t)$ for $t \leq \frac{1}{2}$ and $\chi^{\prime}(y, t)=(y, t)$ for $t \geq \frac{1}{2}$. Then $\chi \cdot \chi^{\prime}$ is represented by $\chi^{\prime \prime}$, where $\chi^{\prime \prime}(y, t)=\chi^{\prime}(y, 2 t)$ for $t \leq \frac{1}{2}$ and $\chi^{\prime \prime}(y, t)=(y, 2 t-1)$ for $t \geq \frac{1}{2}$. Let $\bar{\chi}$ and $\bar{\chi}^{\prime}$ be extensions of $\chi$ and $\chi^{\prime}$ respectively to diffeomorphisms $P_{2 l-1} \times I \rightarrow P_{2 l-1} \times I$; we may assume that $\bar{\chi}(y, t)=(y, t)$ for $t \leq \frac{1}{2}$ and that $\bar{\chi}^{\prime}(y, t)=\left(\varphi^{\prime}(y), t\right)$ for $t \geq \frac{1}{2}$. Clearly $\chi^{\prime \prime}$ is isotopic modulo boundary to $\bar{\chi} \circ \bar{\chi}^{\prime} \mid P_{l-2} \times I$ so that $\bar{\chi} \circ \bar{\chi}^{\prime}$ is a diffeomorphism $P_{2 l-1} \times I \rightarrow P_{2 l-1} \times I$ restricting to a diffeomorphism $P_{2 l-1} \times 1 \rightarrow P_{2 l-1} \times 1$ representing $\partial_{1}\left(x \cdot x^{\prime}\right)$. But clearly $\bar{\chi} \circ \bar{\chi}^{\prime} \mid P_{2 l-1} \times 1=\varphi \circ \varphi^{\prime} \times 1$.

Construction of $\partial_{1}^{\prime}$. The map $\partial_{1}^{\prime}$ is constructed in the same way as $\partial_{1}$. If $x \in \pi_{1} \xi:\left(N, L: P_{2 l-1}\right)$ is represented by the embedding $\chi: N \times I \rightarrow P_{2 l-1} \times I$ constantly the identity near $N \times 0$ and $N \times 1$, let $\bar{\chi}: P_{2 l-1} \times I \rightarrow P_{2 l-1} \times I$ be an extension of $\chi$ to a diffeomorphism the identity near $P_{2 l-1} \times 0$ and level preserving near $P_{2 l-1} \times 1$. Then $\bar{\chi}(y, 1)=(\varphi(y), 1)$ with $\varphi \in \operatorname{Diff}\left(P_{2 l-1}, N\right)$ and we specify that $\varphi$ represents $\partial_{1}^{\prime} x \in \pi_{0}$ Diff: $\left(P_{2 l-1}, N\right)$. The same argument as for $\partial_{1}$ shows that $\partial_{1}^{\prime}$ is a homomorphism.

Lemma 1.1. The two sequences

$$
\pi_{1} \xi:\left(P_{l-2}: P_{2 l-1}\right)_{L} \stackrel{a_{1}}{\rightarrow} \pi_{0} \text { Diff: }\left(P_{2 l-1}, P_{l-2}\right) \rightarrow \pi_{0} \text { Diff: } P_{2 l-1}
$$

and

$$
\pi_{1} \xi:\left(N, L: P_{2 l-1}\right) \stackrel{a_{1}}{\rightarrow} \pi_{0} \text { Diff: }\left(P_{2 l-1}, N\right) \rightarrow \pi_{0} \text { Diff: }\left(P_{2 l-1}, P_{l-2}\right)
$$

are exact.

Proof. Suppose $x \in \operatorname{ker}\left(\pi_{0}\right.$ Diff: $\left(P_{2 l-1}, P_{l-2}\right) \rightarrow \pi_{0}$ Diff: $\left.P_{2 l-1}\right)$. Then $x$ is represented by $\varphi: P_{2 l-1} \rightarrow P_{2 l-1}$, fixed on $P_{-2}$, and there exists a diffeomorphism $\bar{\chi}: P_{2 l-1} \times I \rightarrow P_{2 l-1} \times I$ such that $\bar{\chi}(y, 1)=(\varphi(y), 1)$ and $\bar{\chi}(y, 0)=(y, 0)$. Then the construction of $\partial_{1}$ above applied to $\bar{\chi} \mid P_{-2} \times I=\chi$ shows that $\chi$ represents $z \in \pi_{1} \xi:\left(P_{l-2}, P_{2 l-1}\right)$ such that $\partial_{1} Z=x$. The proof of exactness in the other sequence is the same.

Lemma 1.2. The map $\pi_{0}$ Diff: $\left(P_{2 l-1}, P_{l-2}\right) \rightarrow \pi_{0}$ Diff: $P_{2 l-1}$ is an isomorphism.

Proof. We have that the following sequence is exact:

$$
\pi_{1} \xi:\left(P_{l-2}, P_{2 l-1}\right)_{L} \stackrel{a_{1}}{\rightarrow} \pi_{0} \text { Diff: }\left(P_{2 l-1}, P_{l-2}\right) \rightarrow \pi_{0} \text { Diff: } P_{2 l-1} \rightarrow \pi_{0} \xi:\left(P_{l-2}, P_{2 l-1}\right) \text {. }
$$

There are two homotopy classes of maps $P_{l-2} \rightarrow P_{2 l-1}$. Since $2(l-2)+3$ $\leq 2 l-1$, Whitney's embedding theorem implies that there are exactly two isotopy classes of embeddings $P_{l-2} \rightarrow P_{2 l-1}$. Thus $\pi_{0} \xi:\left(P_{l-2}, P_{2 l-1}\right)$ consists of just 
two elements, and it is clear that the image of the right-most map in the sequence above consists of the isotopy class of embeddings inducing an isomorphism in the fundamental group. Thus the map of the lemma is an epimorphism. To see that it is a monomorphism, we examine the other end of the exact sequence. $\mathbf{A}$ map $f: P_{l-2} \times I \rightarrow P_{2 l-1} \times I$ such that $f(x, t)=(x, t)$ for $t$ near 0 and near 1 induces a map $\bar{f}: P_{l-2} \times s^{1} \rightarrow P_{2 l-1}$. There are two homotopy classes mod $P_{l-2}$ $\times\{0,1\}$ of maps $f$-the class such that $\bar{f}^{*} w=w$ and the class such that $\bar{f}^{*} w=w+\xi$ where $w$ is the generator of $H^{1}\left(P: Z_{2}\right)$ or any of its obvious pullbacks, and $\xi$ is the generator of $H^{1}\left(S^{1}: Z_{2}\right)$ pulled back to $H^{1}\left(P_{l-2} \times S^{1}: Z_{2}\right)$. Since such a map $f$ is already an embedding near $P_{l-2} \times\{0,1\}$, and since $f_{*}: \pi_{i}\left(P_{l-2}\right) \rightarrow \pi_{i}\left(P_{2 l-1}\right)$ is an isomorphism for $i<l-2$, Haefliger's theorem on embeddings implies that to each of the two homotopy classes corresponds exactly one isotopy class. And since they are homotopically distinct, it follows then that to each homotopy class corresponds exactly one concordance class. Thus $\pi_{1} \xi:\left(P_{l-2}: P_{2 l-1}\right)_{L}=Z_{2}$. Let $y \in \pi_{1} \xi:\left(P_{l-2}: P_{2 l-1}\right)_{L}$ be the nontrivial class-it is represented by any embedding $f$ as above such that $\bar{f}^{*} w=w+\xi$. We construct such an embedding: let $g: I \rightarrow S O(2 l)$ be a path such that $g(0)=$ id and $g(1)=-$ id. Then let $f_{0}$ be the composition

$$
I \times P_{l-2} \stackrel{g \times i d}{\longrightarrow} S O(2 l) \times P_{l-2} \dot{\rightarrow} P_{2 l-1}
$$

where $S O(2 l) \times P_{l-2} \rightarrow P_{2 l-1}$ is the restriction of the action $S O(2 l) \times P_{2 l-2}$ $\dot{\rightarrow} P_{2 t-1}$. Then define $f$ by $f(x, t)=\left(f_{0}(t, x), t\right)$. Clearly $f_{0}$ factors through $S^{1}$ $\times P_{l-2}$ :

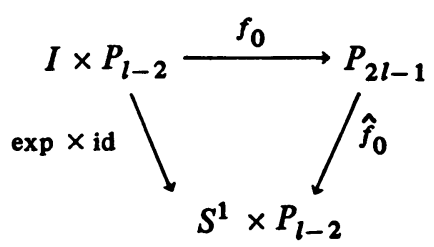

where $\exp t=(\cos 2 \pi t, \sin 2 \pi t)$. To see that $\bar{f}_{0}{ }^{*} w=w+\xi$, it suffices to check that the composition $S^{1} \stackrel{x^{*}}{\longrightarrow} S^{1} \times P_{l-2} \stackrel{f_{0}}{\longrightarrow} P_{2 l-1}$ is homotopically nontrivial. And to check that, it suffices to see that the composition

$$
I \stackrel{\exp }{\longrightarrow} S^{1} \stackrel{x^{*}}{\longrightarrow} S^{1} \times P_{l-2} \rightarrow P_{2 l-1}
$$

lifts to $I \stackrel{h}{\rightarrow} S^{2 l-1}$ with $h(0) \neq h(1)$. But if $\{ \pm x\} \subset S^{l-2} \subset S^{2 l-1}$ is the inverse image of * under the canonical projection $S^{l-2} \rightarrow P_{l-2}$, a lift $h$ of that composition is given by $h(t)=g(t) \cdot x$. Then $h(0)=x \neq-x=h(1)$, and $f$ represents $y$. We extend $f$ to a diffeomorphism $\chi: P_{2 l-1} \rightarrow I \times P_{2 l-1} \times I$ by defining $\chi_{0}$ to be the composition $I \times P_{2 l-1} \stackrel{8 \times 1 d}{\longrightarrow} S O(2 l) \times P_{2 l-1} \rightarrow P_{2 l-1}$ and setting $\chi(x, t)=\left(\chi_{0}(t, x) t\right)$. But then $\chi(x, 1)=(x, 1)$ so that the identity map represents $\partial_{1} y$. Thus 
$\partial_{1}: \pi_{1} \xi:\left(P_{l-2}, P_{2 l-1}\right)_{L} \rightarrow \pi_{0}$ Diff: $\left(P_{2 l-1}, P_{l-2}\right)$ is the trivial homomorphism and the proof of the lemma is complete.

Thus far we have reduced the computation of $\pi_{0}$ Diff: $P_{2 l-1}$ to the computation of $\pi_{0}$ Diff: $\left(P_{2 l-1}, P_{l-2}\right)$. Our next objective is to estimate how closely the group $\pi_{0}$ Diff: $\left(P_{2 l-1}, N\right)$ approximates the group $\pi_{0}$ Diff: $\left(P_{2 l-1}, P_{l-2}\right)$ via the homomorphism $\pi_{0}$ Diff: $\left(P_{2 l-1}, N\right) \rightarrow \pi_{0}$ Diff: $\left(P_{2 l-1}, P_{l-2}\right)$. If $\zeta$ is a vector bundle $|\zeta| \rightarrow X$, let $G L(\zeta)$ be the subfiber bundle of $\operatorname{Hom}(\zeta, \zeta)$ consisting of nonsingular maps. Then the space $\Gamma(G L(\zeta))$ of sections of $G L(\zeta)$ may be interpreted as the space of vector bundle equivalence $\zeta \rightarrow \zeta$. Composition turns $\Gamma(G L(\zeta))$ into an $H$-space so that $\pi_{0} \Gamma(G L(\zeta))$ inherits a group structure and $\pi_{i} \Gamma(G L(\zeta))$ is abelian for $i \geq 1$. Choose an equivalence $\varphi: v\left(P_{l-2}: P_{2 l-1}\right) \rightarrow(l+1) y_{l-2}$ where $y_{r} \rightarrow P_{r}$ is the canonical line bundle. Then define $\bar{\delta}_{0}: \xi\left(N, L: P_{2 l-1}\right) \rightarrow \Gamma\left(G L\left((l+1) y_{l-2}\right)\right)$ by setting $\bar{\delta}_{0} f=\varphi \circ\left(d f \mid v\left(P_{l-2}: P_{2 l-1}\right)\right) \circ \varphi^{-1}$. For any spaces $X$ and $Y$ let $[X: Y]$ be the space of continuous maps $X \rightarrow Y$. Define $\bar{\delta}_{1}: \xi\left(N \times I, j_{L}: P_{2 l-1} \times I\right) \rightarrow[I$ : $\left.\Gamma\left(G L\left((l+1) y_{t-2}\right)\right)\right]$ by setting $\bar{\delta}_{1} F(t)(x)=\varphi \circ d \mathrm{pr} \circ d F\left(\varphi^{-1}(x), t\right)$ where $t \in I$, $x \in(l+1) y_{l-2}$, and $v\left(P_{l-2} \times I: P_{2 l-1} \times I\right)=v\left(P_{l-2}: P_{2 l-1}\right) \times I$, and pr: $P_{2 l-1} \times I$ $\rightarrow P_{2 l-1}$ is the projection. Clearly $\bar{\delta}_{1} F(0)=\bar{\delta}_{1} F(1)=\bar{\delta}_{0}$ (id) so that $\bar{\delta}_{1}$ factors through the loop space to define

$$
\bar{\delta}_{1}: \xi\left(N \times I, J_{L}: P_{2 l-1} \times I\right) \rightarrow \Omega\left(\Gamma\left(G L\left((l+1) y_{l-2}\right)\right)\right) .
$$

Clearly $\bar{\delta}_{0}$ and $\bar{\delta}_{1}$ induce maps

$$
\delta_{0}: \pi_{0} \xi:\left(N, L: P_{2 l-1}\right) \rightarrow \pi_{0}\left(G L\left((l+1) y_{l-2}\right)\right)
$$

and

$$
\delta_{1}: \pi_{1} \xi:\left(N, L: P_{2 l-1}\right) \rightarrow \pi_{0} \Omega\left(\Gamma\left(G L(l+1) y_{l-2}\right)\right) .
$$

Lemma 1.3. $\delta_{0}$ and $\delta_{1}$ are isomorphisms.

Proof. We leave to the reader the proof that $\delta_{1}$ is a homomorphism, and the definition of a group structure on $\pi_{0} \delta:\left(N, L: P_{2 l-1}\right)$ such that $\delta_{0}$ is a homomorphism. By Hirsch's immersion theorem or by the tubular neighborhood theorem, both maps are onto with trivial kernel, and the lemma is proved.

Now, there is a homomorphism

$$
\Omega \Gamma\left(G L\left((l+1) y_{l-2}\right)\right) \rightarrow \Gamma^{\prime}\left(G L\left((l+1) y_{l-2} \times S^{1}\right)\right)
$$

where $\Gamma^{\prime}$ denotes the space of sections the identity over $P_{l-2} \times 1$. And as in [10], there exist isomorphisms

$$
\pi_{0} \Gamma\left(G L\left((l+1) y_{l-2}\right)\right) \stackrel{\cong}{\rightrightarrows} \pi_{0}\left[P_{l-2}: G L(R, \infty)\right]
$$

and

$$
\pi_{0} \Gamma^{\prime}\left(G L\left((l+1) y_{l-2} \times S^{1}\right)\right) \cong \pi_{0}\left[P_{l-2} \times S^{1 / P_{l-2}} \times 1: S L(R, \infty)\right]
$$


where we write $S L(R, \infty)$ in the second case since $P_{l-2} \times 1 \rightarrow$ id. Finally, the two right-hand groups above are, respectively,

$$
\begin{aligned}
Z_{2}+(K O)^{\sim-1}\left(P_{l-2}\right) & =Z_{2}+Z_{2} \text { and } \\
(K O)^{\sim-1}\left(S P_{l-2}\right)+(K O)^{\sim-1}\left(S^{1}\right) & =Z_{2}+Z_{2} .
\end{aligned}
$$

We are now assuming $l \equiv 6 \bmod 8$; the summand $Z_{2}$ in the first group is introduced by the sign-determinant homomorphism $G L(R, \infty) \rightarrow Z_{2}$.

Lemma 1.4. Let $l \equiv 6 \bmod 8$. Then the image of the homomorphism $\pi_{0}$ Diff: $\left(P_{2 l-1}, P_{l-2}\right) \stackrel{\partial_{0}}{\longrightarrow} \pi_{0} \xi:\left(N, L: P_{2 l-1}\right)$ is a group of order two, and the image splits back into $\pi_{0}$ Diff: $\left(P_{2 l-1}, P_{l-2}\right)$.

Proof. The diffeomorphism

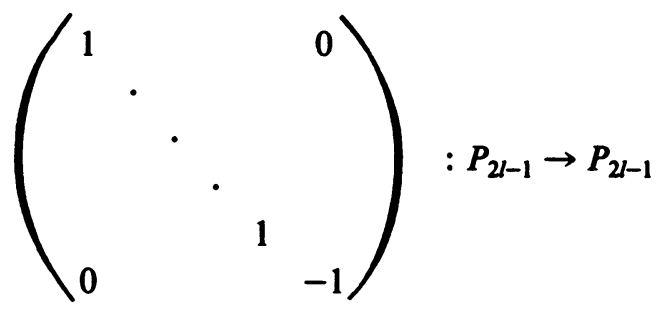

is the identity on $P_{l-2}$ so that it defines an element $\zeta \in \pi_{0}$ Diff: $\left(P_{2 l-1}, P_{l-2}\right)$. Clearly $\zeta^{2}=1$. But $\delta_{0}\left(\partial_{0}(\zeta)\right)$ is represented as a vector bundle equivalence by $y_{l-2} \oplus l y_{l-2} \stackrel{-1 \oplus 1}{\longrightarrow} y_{l-2} \oplus l y_{l-2}$. The map of [10] from $\pi_{0} G L(\xi)$ to $\pi_{0}[X: G L(R, \infty)]$, for $\xi \rightarrow X$ a vector bundle over a finite CW complex, is defined as follows: Let $\xi^{\prime}$ be a vector bundle inverse to $\xi$ and $E: \xi \oplus \xi^{\prime} \rightarrow X \times R^{n}$ a vector bundle equivalence. If $\gamma: \xi \rightarrow \xi$ is an equivalence, then $E \circ(\gamma \oplus$ id $) \circ E^{-1}$ is given by a map $X \rightarrow G L(R, n)$. We carry the class of $\gamma$ to the class of the composition $X \rightarrow G L(R, n) \rightarrow G L(R, \infty)$. Since $(K O)^{-1}\left(P_{l-2}\right) \rightarrow(K O)^{-1}\left(P_{1}\right)$ is an isomorphism, it follows that we have a commutative diagram of isomorphisms

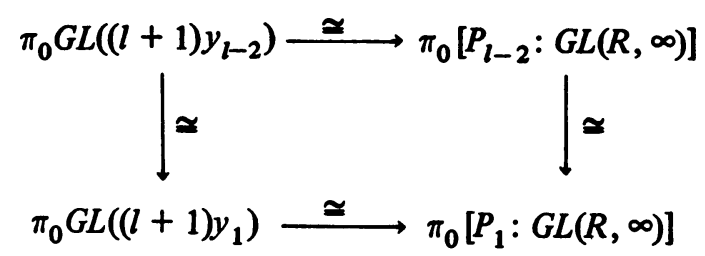

On the other hand it is easy to check that the equivalence $-1: y_{1} \rightarrow y_{1}$ is carried by the isomorphism $\pi_{0} G L\left(y_{1}\right) \cong \pi_{0}\left[P_{1}: G L(R, \infty)\right]=Z_{2}+(K O)^{\sim-1}\left(P_{1}\right)$ to the element $x_{1}$, where $x_{1}$ is the generator of $Z_{2}$ and $x_{2}$ the generator of $(K O)^{-1}\left(P_{1}\right)$. Since $x_{1}$ has order two, we have that the image of $\partial_{0}$ contains $x_{1}$, which splits back into $\pi_{0}$ Diff: $\left(P_{2 l-1}, P_{l-2}\right)$ via $x_{1} \rightarrow \zeta$.

It remains to show that the image of $\partial_{0}$ is the group generated by $x_{1}$; it will suffice to show that $\partial_{0}$ is not an epimorphism. Let $\varphi: P_{2 l-1} \rightarrow P_{2 l-1}$ be a 
diffeomorphism fixed on $P_{1}$ such that $\partial_{0} \varphi=x_{2}$. Let $P_{l-1}$ and $P_{l-1}^{\prime}$ be two standard linking copies of $P_{l-1}$ in $P_{2 l-1}$, with $P_{1} \subset P_{-1}$. By Haefliger's embedding theorem, we may isotope $\varphi$ modulo $P_{1}$ so that $\varphi\left|P_{l-1}=\mathrm{id}\right| P_{l-1}$ and $\varphi\left|P_{l-1}^{\prime}=\mathrm{id}\right| \boldsymbol{P}_{l-1}^{\prime}$. We may assume that $\varphi$ carries tubular neighborhoods of $P_{l-1}$ and $P_{l-1}^{\prime}$ linearly onto themselves. Let $P_{1}^{\prime}$ be a copy of $P_{1}$ in $P_{l-1}^{\prime}$, and let $X$ be the manifold $I \times P_{2 l-1}$ collapsed by $(0, x) \sim(1, \varphi(x))$. Then we have two embeddings $S^{1} \times P_{1}$ $\subset X$ and $S^{1} \times P_{1} \stackrel{\cong}{\rightarrow} S^{1} \times P_{1} \subset X$ which induce the same map on fundamental groups. The normal bundle of the first is a sum of canonical line bundles plus a nontrivial bundle pulled back from $S^{2}$ via $S^{1} \times P_{1} \rightarrow S^{2}$; this nontrivial additional bundle arises from the fact that $d \varphi: v\left(P_{1}: P_{2 l-1}\right) \rightarrow v\left(P_{1}: P_{2 l-1}\right)$ is nontrivial. If $d \varphi: v\left(P_{1}^{\prime}: P_{2 l-1}\right) \rightarrow v\left(P_{1}^{\prime}: P_{2 l-1}\right)$ were trivial, then the normal bundle of the second embedding would be a sum of canonical line bundles and the two bundles would be inequivalent. But there is only one isotopy class of embeddings $S^{1} \times P_{1} \rightarrow X$ inducing a given map on fundamental group; thus the two bundles are equivalent and we must have that $d \varphi: v\left(P_{1}^{\prime}: P_{2 l-1}\right) \rightarrow v\left(P_{1}^{\prime}: P_{2 l-1}\right)$ is nontrivial. Now it follows that the two bundle maps $v\left(P_{l-1}: P_{2 l-1}\right) \stackrel{\beta}{\rightarrow} v\left(P_{l-1}: P_{2 l-1}\right)$ and $v\left(P_{l-1}^{\prime}: P_{2 l-1}\right) \stackrel{\beta}{\rightarrow} v\left(P_{l-1}^{\prime}: P_{2 l-1}\right)$ defined by $d \varphi$ are nontrivial. Realizing $v\left(P_{l-1}:\right.$ $\left.P_{2 l-1}\right)$ as $S^{l-1} \times{ }_{z_{2}} R^{l}$, where $Z_{2}$ acts on $S^{l-1} \times R^{l}$ by $(x, y) \rightarrow(-x,-y)$, it is straightforward to check that the bundle map $\alpha: v\left(P_{l-1}: P_{2 l-1}\right) \rightarrow v\left(R_{-1}: P_{2 l-1}\right)$ defined by $\alpha[x, y]=[x, T(y-2(y \cdot x) x)]$ determines the nontrivial element of $(K O)^{-1}\left(P_{-1}\right)$, where [ ] denotes the equivalence class with respect to the $Z_{2}$ action and $T: R^{l} \rightarrow R^{l}$ is the map $T\left(x_{1}, \ldots, x_{l}\right)=\left(-x_{1}, x_{2}, \ldots, x_{l}\right)$. It follows that there exists a map $\left(P_{l-1}, P_{l-2}\right) \stackrel{8}{\rightarrow}(S O(l)$, id $)$ such that the induced map $S^{l-1} \stackrel{\delta}{\rightarrow} S O(l)$ represents an element of $\operatorname{ker}\left(\pi_{l-1}(S O(l)) \rightarrow \pi_{l-1}(S O(l+1))\right)$ and such that $\beta=\alpha \circ \gamma$ where $\gamma: v\left(P_{l-1}: P_{2 l-1}\right) \rightarrow v\left(P_{l-1}: P_{2 l-1}\right)$ is the map, identity over $\boldsymbol{P}_{l-2}$, determined by $g$. Similarly, we have $\alpha^{\prime}: v\left(P_{l-1}: P_{2 l-1}\right) \rightarrow v\left(R_{-1}: P_{2 l-1}\right)$ defined by $\alpha^{\prime}[x, y)=T(x-2(x \cdot y) y, y)$ and $\left(P_{l-1}^{\prime}, P_{l-2}^{\prime}\right) \stackrel{g^{\prime}}{\rightarrow}(S O(l)$, id $)$ such that $\beta^{\prime}=\alpha^{\prime} \circ \gamma^{\prime}$. By isotoping $\varphi$, we may assume that there is a covering diffeomorphism $\bar{\varphi}: S^{2 l-1} \rightarrow S^{2 l-1}$ such that $\bar{\varphi}$ carries tubular equivariant neighborhoods of $S^{l-1}$ and $S^{\prime-1}$ onto themselves. Then on the copy of $S^{l-1} \times S^{l-1} \times I$ between the two tubular neighborhoods, $\bar{\varphi}$ defines a homotopy between $f_{0}$ : $S^{l-1} \times S^{l-1} \rightarrow S^{l-1} \times S^{l-1}$ and $f_{1}: S^{l-1} \times S^{l-1} \rightarrow S^{l-1} \times S^{l-1}$, where

$$
f_{0}(x, y)=\left(x, \bar{g}(x)^{2} \cdot T(y-2(x \cdot y) x)\right)
$$

and

$$
f_{1}(x, y)=\left(\bar{g}^{\prime}(y)^{2} \cdot T(x-2(x \cdot y) y), y\right) .
$$

But $f_{0 *}: H_{l-1}\left(S^{l-1} \times S^{l-1}: Z_{4}\right) \rightarrow H_{l-1}\left(S^{l-1} \times S^{l-1}: Z_{4}\right)$ is represented by the matrix $\left(\begin{array}{ll}1 & 2 \\ 0 & 1\end{array}\right)$ and $f_{1 *}$ by $\left(\begin{array}{ll}1 & 0 \\ 2 & 1\end{array}\right)$ so that $f_{0}$ and $f_{1}$ cannot be homotopic. Thus we have arrived at a contradiction, and the diffeomorphism $\varphi$ cannot exist. Lemma 4 is now proved. 
If $M$ is orientable, let $\operatorname{Diff}^{+}(M, X)$ be the orientation-preserving diffeomorphisms fixing $X$. As above we obtain an isomorphism $\pi_{0}$ Diff $^{+}:\left(P_{2 l-1}, P_{l-2}\right)$ $\rightarrow \pi_{0}$ Diff $^{+}: P_{2 l-1}$. Let $s: \pi_{0}$ Diff: $P_{2 l-1} \rightarrow Z_{2}$ be the homomorphism defined by $s(\varphi)=1$ for $\varphi$ orientation reversing and $s(\varphi)=0$ for $\varphi$ orientation preserving. Then Lemma 1.4 becomes the statement that

$$
1 \rightarrow \pi_{0} \text { Diff }^{+}: P_{2 l-1} \rightarrow \pi_{0} \text { Diff: } P_{2 l-1} \stackrel{s}{\rightleftarrows} Z_{2} \rightarrow 0
$$

is split exact, and

(2) the obvious homomorphism $\pi_{0}$ Diff $^{+}:\left(P_{2 l-1}, P_{l-2}\right) \rightarrow(K O)^{-1}\left(P_{l-2}\right)$ is trivial.

From the first of these we see that $\pi_{0}$ Diff: $P_{2 l-1}$ is the semidirect product of $\pi_{0}$ Diff $^{+}: P_{2 l-1}$ with $Z_{2}$, the action of $Z_{2}$ on $\pi_{0}$ Diff $^{+}: P_{2 l-1}$ being defined by $0 \cdot \varphi=\varphi$ and $1 \cdot \varphi=\zeta \circ \varphi \circ \zeta$ where $\zeta$ is the diffeomorphism in the proof of Lemma 1.4.

2. The bordism mapping torus. We are seeking to identify the group $\pi_{0}$ Diff $^{+}$: $P_{2 l-1}$ with a stable homotopy group for $l \equiv 6 \bmod 8$. It follows from the preceding section that $\pi_{0}$ Diff $^{+}:\left(P_{2 l-1}, P_{l-2}\right)$ is isomorphic to $\pi_{0}$ Diff $^{+}: P_{2 l-1}$ so it suffices to find an isomorphism of $\pi_{0}$ Diff $^{+}:\left(P_{2 l-1}, P_{l-2}\right)$ with a stable homotopy group. The stable homotopy group will be a Lashof cobordism group $\Omega_{21}(v)$ and the isomorphism $t: \pi_{0}$ Diff $^{+}:\left(P_{2 l-1}, P_{l-2}\right) \rightarrow \Omega_{2 l}(v)$ will be a bordism variation on the mapping torus construction.

We begin by constructing a Lashof cobordism theory. Let $P_{\infty} \rightarrow B S O$ be a classifying map for an orientation of the vector bundle $k y_{\infty}$ where $k=d 2^{L}-2 l$ with $L \geq \varphi(l)$ and $d 2^{L} \geq 2$. Let $P_{\infty} \rightarrow P[l] \stackrel{\leftrightarrow}{\rightarrow} B S O$ be the lth stage in the Moore-Postnikov decomposition of that map. Let $F$ be the fiber of $P_{\infty} \rightarrow B S O$; we will have a commutative diagram

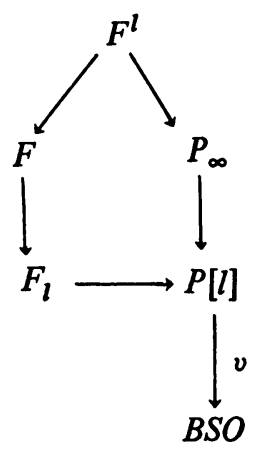

where $F_{l}$ is the fiber of $v$ and $F^{l}$ the fiber of both $F \rightarrow F_{l}$ and $P[l] \stackrel{\bullet}{\longrightarrow} B S O$; we will have $\pi_{i}\left(F^{l}\right)=0$ for $i \leq l$ and $\pi_{i}\left(F^{\prime}\right) \stackrel{\cong}{\rightrightarrows} \pi_{i}(F)$ for $i>l$. Then $\pi_{i}(F)$ $\stackrel{\rightrightarrows}{\rightarrow} \pi_{i}\left(F_{l}\right)$ for $i \subseteq l$ and $\pi_{i}\left(F_{l}\right)=0$ for $i>l$. Now we use the hypothesis that $l \equiv 6 \bmod 8: S O$ is a double cover of $F$. Since $\pi_{l}(S O)=\pi_{l-1}(S O)=0$, it 
follows that $\pi_{i}\left(F_{l}\right)=0$ for $i \geq l-1$. The fibration $v$ defines as in [6] a Lashof cobordism theory $\Omega_{*}(v)$.

Next we define the map $t: \pi_{0}^{+}$Diff: $\left(P_{2 l-1}, P_{l-2}\right) \rightarrow \Omega_{2 l}(v)$. If

$$
\varphi \in \operatorname{Diff}\left(P_{2 l-1}, P_{l-2}\right),
$$

let $\bar{\varphi}: P_{2 l-l} \times R \rightarrow P_{2 l-1} \times R$ be defined by $\bar{\varphi}(x, t)=(\varphi(x), t+1)$. Then $\bar{\varphi}$ is the generator of a smooth free action of $Z$ on $P_{2 l-1} \times R$. Denote the orbit manifold by $T(\varphi)$ - of course $T(\varphi)$ is just the usual mapping torus. Since $\varphi \mid P_{l-2}=$ id, we have a distinguished embedding $P_{l-2} \times S^{1} \subset T(\varphi)$; also we have $P_{2 l-1} \subset T(\varphi)$ induced by $P_{2 l-1} \times 0 \subset P_{2 l-1} \times R$. Then $P_{l-2} \times S^{1} \cap P_{2 l-1}=P_{l-2} \times 1$ so that the two maps $P_{l-2} \times S^{1} \rightarrow P_{l-2} \subset P_{2 l-1} \subset P_{\infty}$ and $P_{2 l-1} \subset P_{\infty}$ agree to define a map $P_{l-2} \times S^{1} \cup P_{2 l-1} \stackrel{L}{\longrightarrow} P_{\infty}$. Notice that the composition $v_{0}: P_{l-2} \times S^{1} \cup P_{2 l-1}$ $\stackrel{\iota}{\longrightarrow} \rightarrow$ BSO classifies an orientation of the stable normal bundle of $T(\varphi)$ restricted to $P_{l-2} \times S^{1} \cup P_{2 l-1}$. It follows that there exists a map $\bar{v}(\varphi): T(\varphi)$ $\rightarrow B S O$ classifying that orientation of the stable normal bundle, and extending $v_{0}$. Thus we have the following commuative diagram

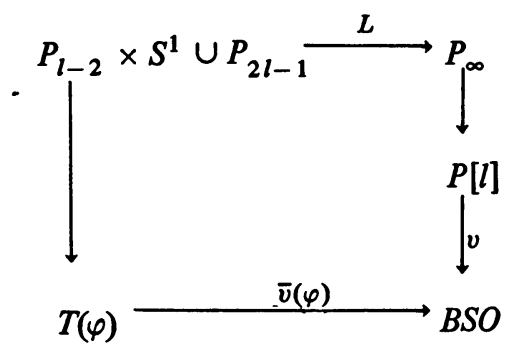

We wish to find a lift $v(\varphi): T(\varphi) \rightarrow P[l]$ which will keep the diagram commutative.

Since $\pi_{1}\left(P_{l-2} \times S^{1} \cup P_{2 l-1}\right) \rightarrow \pi_{1}(T(\varphi))$ is an isomorphism and since by restricting the cover $P_{2 l-1} \times R \rightarrow T(\varphi)$ to $P_{l-2} \times S^{1} \cup P_{2 l-1}$ we obtain $P_{l-2} \times R$ $\cup P_{2 l-1} \times Z \subset P_{2 l-1} \times R$, we see that $P_{l-2} \times S^{1} \cup P_{2 l-1}$ is a $\mathrm{CW}(l-1)$ skeleton of $T(\varphi)$. It follows that

$$
H^{j+1}\left(T(\varphi), P_{l-2} \times S^{1} \cup P_{2 l-1}: \pi_{j}\left(F_{l}\right)\right)=0
$$

for $j+1 \leq l-1$. But this group is 0 also for $j \geq l-1$, since $\pi_{j}\left(F_{l}\right)=0$ for $j \geq l-1$, so that there is no obstruction to the existence of $v(\varphi)$. Similarly $H^{j}\left(T(\varphi), P_{l-2} \times S^{1} \cup P_{2 l-1}: \pi_{j}\left(F_{l}\right)\right)=0$ for $j \leq l-1$ since $P_{l-2} \times S^{1} \cup P_{2 l-1}$ is an $(l-1)$ skeleton, but that group is also zero for $j \geq l-1$ since $\pi_{j}\left(F_{l}\right)=0$ then. Consequently, there is a unique isotopy class (= bundle homotopy class mod $\left.P_{l-2} \times S^{1} \cup P_{2 l-1}\right)$ of lifts $v(\varphi)$ of $\bar{v}(\varphi)$.

We define $t(\varphi)$ by $t(\varphi)=$ cobordism class of $v(\varphi)$. That $t(\varphi)$ is well defined follows from the fact that $v(\varphi)$ depends uniquely on $\bar{v}(\varphi)$-a homotopy between two choices $\bar{v}_{0}(\varphi)$ and $\bar{v}_{1}(\varphi)$ of $\bar{v}(\varphi)$ lifts to a homotopy from $v_{0}(\varphi)$ to $v_{1}^{\prime}(\varphi)$ with 
$v_{1}^{\prime}(\varphi)$ isotopic to $v_{1}(\varphi)$ over $\bar{v}_{1}(\varphi)$. To see that $t$ is a homomorphism, let $e(\varphi): P_{2 l-1} \times[-1,1] \subset T(\varphi)$ be an embedding such that $e(\varphi): P_{2 l-1} \times 0 \subset T(\varphi)$ is just the embedding $P_{2 l-1} \subset T(\varphi)$ above. Let

$$
X=(T(\varphi) \times I)+\left(P_{2 l-1} \times[-1,1] \times I\right)+(T(\varphi) \times I) / \sim
$$

where $(e(\varphi)(x), 1) \sim(x, 0)$ for $x \in P_{21-1} \times[-1,1]$ and $(x, 1) \sim(e(\psi)(x), 1)$. Define $X \stackrel{v(\varphi, t)}{\longrightarrow} P[l]$ by means of $T(\varphi) \times 1 \stackrel{v(\varphi)}{\longrightarrow} P[l], P_{2 l-1} \times 0 \times I \rightarrow P_{2 l-1}$ $\stackrel{v(\varphi)-v(\psi)}{\longrightarrow} P[l]$ and $T(\psi) \times 1 \stackrel{v(\psi)}{\longrightarrow} P[l]$ and a retraction $X \rightarrow T(\varphi) \times 1 \cup P_{2 l-1}$ $\times 0 \times I \cup T(\psi) \times 1$. Then $v(\varphi, \psi)$ determines a cobordism from $v(\varphi)+v(\psi)$ to $v(\varphi \circ \psi)$. Finally, a concordance $\bmod P_{l-2}$ from $\varphi$ to $\psi$ gives rise to a cobordism from $v(\varphi)$ to $v(\psi)$ by means of constructions like those above, so that finally $t$ factors through $\pi_{0}$ Diff $^{+}:\left(P_{2 l-1}, P_{l-2}\right)$ to define a homomorphism $t: \pi_{0}$ Diff $^{+}$: $\left(P_{2 l-1}, P_{l-2}\right) \rightarrow \Omega_{2 l}(v)$.

Remark. Let $\zeta$ be the orientation reversing diffeomorphism in Lemma 1.4. Since $\zeta^{2}=$ id, the following diagram commutes:

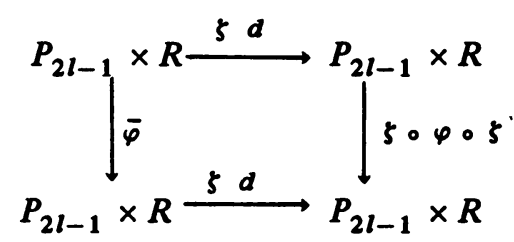

a diffeomorphism $\lambda: T(\varphi) \rightarrow T(\zeta \circ \varphi \circ \zeta)$ making the following diagram commute:

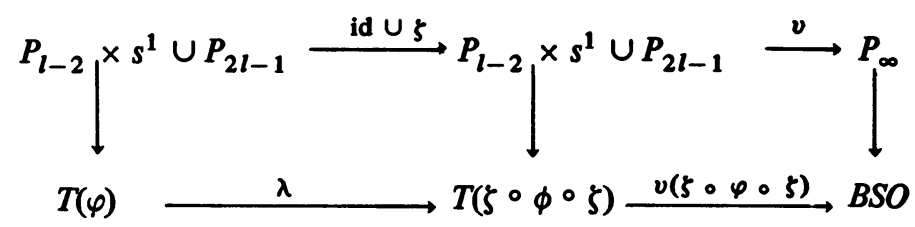

Let $v(\zeta \circ \varphi \circ \zeta)$ be the lift defined by the right square and $v^{\prime}(\varphi)$ the lift defined by the outer square. Then clearly $v^{\prime}(\varphi)=v(\zeta \circ \varphi \circ \zeta) \circ \lambda$ so $v^{\prime}(\varphi)$ and $v(\zeta \circ \varphi$ $\circ \zeta$ ) define the same element of $\Omega_{2 \lambda}(v)$. But a straightforward argument shows that the element of $\Omega_{21}(v)$ defined by $v^{\prime}(\varphi)$ is the negative of that defined by $v(\varphi)$. Consequently we have the formula

$$
t(\zeta \circ \varphi \circ \zeta)=-t(\varphi)
$$

\section{Proposition 1. $t$ is a monomorphism.}

Proof. Suppose $t(\varphi)=0$. Then there is a $2 l+1$ manifold $X$ with $\partial X=T(\varphi)$ and a normal map $X \rightarrow P[l]$ extending $v(\varphi)$. By means of low dimensional surgery, we may assume that $\pi_{1}(X) \stackrel{\approx}{\rightarrow} P[l]$ and $\pi_{i}(X)=0$ for $i \leq l-1$. It 
follows that there is no obstruction to extending the embedding $P_{-2} \times S^{1} \subset \partial X$ to a map $P_{-2} \times D^{2} \rightarrow X$. But the map $R_{-2} \times D^{2} \rightarrow X$ may be assumed to embed a boundary collar compatibly in a boundary collar of $X$. Since the map induces isomorphisms in $\pi_{i}$ for $i \leq l-3$, Haefliger's embedding theorem applies and we have an embedding $P_{l_{2}} \times D^{2} \subset X$ extending the embedding $P_{-2} \times S^{1} \subset \partial X$. Let $N^{\prime}$ be a tubular neighborhood of $P_{-2} \times D^{2}$ in $X$ which agrees with a collared extension into $X$ of a tubular neighborhood of $P_{-2} \times S^{1}$ in $X$. Let $Y$ be the closure of $X-N^{\prime}$, rounded corners. Now we argue as in [8] or H. Schneider [5].

Lemma 2.1. If $A$ is a finitely generated $Z\left[Z_{2}\right]$-module then $H^{j}(Y ; A)=0$ for $j \geq l+2$.

Proof. $H_{i}(Y, \partial Y ; A)=H_{i}\left(X, \partial X \cup P_{-2} \times D^{2} ; A\right)$. Clearly

$$
\pi_{i}\left(\partial X, P_{l-2} \times S^{1} \cup P_{2 l-1}\right)=0
$$

for $i \leq l-1$. It follows that $H_{i}\left(\partial X \cup P_{-2} \times D^{2} \cup P_{2 l-1}\right)=0$ for $i \leq l-1$. We may conclude from the Hurewicz theorem that $\pi_{i}\left(\partial X \cup R_{-2} \times D^{2}, R_{-2} \times D^{2}\right.$ $\left.\cup P_{2 l-1}\right)=0$ for $i \leq l-1$. Since $P_{l-2} \times D^{2} \cap P_{2 l-1}=P_{l-2}$, we have $\pi_{i}(\partial X$ $\left.\cup P_{l-2} \times D^{2}, P_{2 l-1}\right)=0$ for $i \leq l-1$. The homotopy sequence for a triple now yields $\pi_{i}\left(X, P_{2 l-1}\right) \stackrel{\varkappa_{i}}{\longrightarrow}\left(X, \partial X \cup P_{l-2} \times D^{2}\right)$ an isomorphism for $i \leq l-1$. But $\pi_{i}(X)=\pi_{i-1}\left(P_{2 l-1}\right)=0$ for $i \leq l-1$ so $\pi_{i}\left(X, \partial X \cup P_{-2} \times D^{2}\right)=0$ for $i \leq l$ -1 . Now we see that $H_{i}(Y, \partial Y ; A)=0$ for $i \leq l-1$. And then Poincaré duality implies that $H^{j}(Y ; A)=0$ for $j \geq l+2$, and the lemma is proved.

Let $Y \rightarrow P[l]$ be the restriction to $Y$ of the map $X \rightarrow P[l]$. Since the fibers of $P_{l+1} \rightarrow P_{\infty}$ and $P_{\infty} \rightarrow P[l]$ are $l$-connected, Lemma 2.1 implies that there are no obstructions to lifting $Y \rightarrow P[l]$ to $Y \leftrightarrow P_{l+1}$. The latter map is a normal map since the former is one; notice that $\pi_{i}(Y) \rightarrow \pi_{i}\left(P_{t+1}\right)$ is an isomorphism for $i \leq l-1$.

The map $Y \stackrel{a}{\rightarrow} P_{l+1}$ is covered by a bundle map $A: v\left(Y: R_{+}^{n+2 l+1}\right) \rightarrow k y_{l+1}$ $+(n-k)$ for $n$ large. Let $\alpha$ be the transverse to $* \in P_{1+1}$. Then the framing of $v\left(\{*\}: P_{1+1}\right)$ defines a framing of $v\left(\alpha^{-1}(*): Y\right)$. Write $\alpha^{-1}(*)=(V, \partial V) \subset(Y, \partial Y)$ with the framing given by $v(V: Y) \stackrel{d a}{\longrightarrow} v\left(\{*\}: P_{l+1}\right)$. We consider $\partial Y$ first. The mapping

$$
A \oplus d \alpha: v\left(\partial Y: R^{n+2 l}\right)\left|\partial V \oplus v(\partial V: \partial Y) \rightarrow\left(k y_{l+1}+(n-k)\right)\right|_{*} \oplus(l+1)
$$

gives us, via the Thom-Pontrjagin construction, an element of $\pi_{n+21}\left(S^{n+l+1}\right)$ $=\pi_{l-1}^{s}$. The methods of [11] apply to this situation with little change to show that if that element is zero, then $\alpha \mid \partial V$ may be homotoped so that $\partial V=\varnothing$. But the extension of the bundle map above to

$$
A \oplus d \alpha:\left.\left(v\left(Y: R_{+}^{n+2 l+1}\right) \mid V\right) \oplus(v(V: Y)) \rightarrow\left(k y_{l+1}+(n-k)\right)\right|_{*} \oplus(l+1)
$$

shows that the element in question is actually zero, so we may assume $\partial V=\varnothing$. 
Assuming $\partial V=\varnothing$ now, we may apply the method of [11] again to realize lower dimensional surgeries of $V$ via homotopies of $\alpha$. It follows that we may assume $V$ is $(l-1)$-connected. Since now $V$ is a closed $2 l$-manifold, $H_{i}(V)=0$ for $i \neq 0, l / 2, l$ and $H_{l / 2}(V)$ is a free finitely generated abelian group. Let $T$ be a tubular neighborhood of $V$. We may assume $\varphi:(\operatorname{clos}(Y-T), \partial T) \rightarrow\left(\operatorname{clos}\left(P_{l+1}\right.\right.$ $-D), S$ ) where clos indicates closure, $D$ is a disk neighborhood of $*$ in $P_{l+1}$ and $S$ is its boundary sphere. Clearly $H_{i}(\partial T)=0$ for $i \neq 0, l / 2, l, 3 l / 2,2$ and $H_{l / 2}(\partial T)=H_{3 l / 2}(\partial T)=H_{l / 2}(V)$ and $H_{l}(\partial T)=Z+Z$. We may represent one generator $x$ of $H_{l}(\partial T)$ by a fiber $S^{l} \subset \partial T$ of $\partial T \rightarrow V$ and the other generator $y$ by the image of a cross section $V \rightarrow \partial T$ with trivial normal bundle. Then we have $x \cdot x=y \cdot y=0$ and $x \cdot y=1$, and $\beta_{*}(y)=0$ where $\beta: \partial T \rightarrow S$ is $\alpha \mid \partial T$. We may attach rank $H_{l / 2}(V)$ handles of degree $l / 2+1$ to $\operatorname{clos}(Y-T)$ along $\partial T$ to obtain a manifold $Y_{1}$ and an extension of $\alpha \mid \operatorname{clos}(Y-T)$ to a normal map $\left(Y_{1}, \partial_{1} Y_{1}\right) \stackrel{a_{1}}{\longrightarrow}\left(\operatorname{clos}\left(P_{l+1}-D\right), S\right)$ with $Y_{1}=\partial Y+\partial_{1} Y_{1}$ and $\partial_{1} Y_{1}$ the result of surgering a basis of $H_{l / 2}(\partial T)$. Now we have $H_{i}\left(\partial_{1} Y_{1}\right)=0$ for $i \neq 0, l, 2 l$ and $H_{l}\left(\partial_{l} Y_{l}\right)=Z+Z$ with generators $x_{1}$ and $y_{1}$ such that $x_{1} \cdot x_{1}=y_{1} \cdot y_{1}=0$ and $x_{1} \cdot y_{1}=1$ and $\beta_{*}\left(y_{1}\right)=0$ where $\beta=\alpha_{1} \mid \partial_{1} Y_{1}$. This time $y_{1}$ may be represented by an embedded $l$ sphere with trivial normal bundle, so it may be surgered by adding a handle of degree $l+1$ to $Y_{1}$ along $\partial_{1} Y_{1}$ to obtain $Y_{2}$. Then we obtain an extension of $\alpha_{1}$ to a normal map $\left(Y_{2}, \partial_{1} Y_{2}\right) \rightarrow\left(\operatorname{clos}\left(P_{1+1}-D\right), S\right)$ where $\partial Y_{2}$ $=\partial Y+\partial_{1} Y_{2}$ and $\partial_{1} Y_{2}$ is a homotopy sphere. What homotopy sphere? Since $\partial_{1} Y_{2}=\partial Q$ where $Q$ is the manifold

$$
T \cup\left(\operatorname{rk}\left(H_{l / 2}(V)\right) \times D^{l / 2+1} \times D^{l / 2}\right) \cup D^{l+1} \times D^{l},
$$

an odd dimensional parallelizable manifold, we see by Kervaire-Milnor [2] that $\partial_{1} Y_{2}$ must be the standard homotopy sphere. But then by drilling a hole from $\partial Y$ in $Y_{2}$ to $\partial_{1} Y_{2}$ and restricting $\alpha_{2}$ we obtain $Y_{3}$ and a normal map $\alpha_{3}: Y_{3} \rightarrow P_{1}$. Notice that $\partial Y_{3} \cong \partial Y \# S^{2 l} \cong \partial Y$.

Lemma 2.2. There is a closed manifold $M$, homotopy equivalent to $S^{\prime} \times S^{\prime}$ and a smooth free involution $M \stackrel{\zeta}{\rightarrow}$ such that $M / \zeta \cong \partial Y$.

Proof. By the Seifert-Van Kampen theorem, $\pi_{1}(\partial Y)=Z_{2}$ and $\pi_{1}(\partial Y) \rightarrow \pi_{1}(Y)$ is an isomorphism. We have seen in the proof of Lemma 2.1 that $H_{i}(Y, \partial Y ; A)$ $=0$ for $i \leq l-1$ and $A$ any finitely generated $Z\left[Z_{2}\right]$-module. It follows that $\pi_{i}(Y, \partial Y)=0$ for $i \leq l-1$. Consequently $\pi_{i}(\partial Y)=0$ for $1<i \leq l-2$. Notice that since $\varphi \mid P_{l-2}=$ id, we may isotopy $\varphi \bmod P_{l-2}$ (without changing $t(\varphi)$ or $T(\varphi)$ or $X$ or $Y$ ) so that $\varphi: N \cong N$ where $N$ is a tubular neighborhood of $P_{l-2}$ in $P_{2 l-1}$. Identifying $E\left((l-1) y_{l}\right)$ with the closed complement of $N$ we have $\partial Y=\left(E\left((l-1) y_{l}\right) \times R / \zeta(\varphi)\right) \cup S\left((l+1) y_{l-2}\right) \times D^{2}$, where $S\left((l+1) y_{l-2}\right)$ is identified with $\partial N$. Then the double cover $\overline{\partial Y}$ decomposes, $\overline{\partial Y}=\left(D^{l-l} \times S^{l}\right.$ $\left.\times R / \bar{\varphi}^{\prime}\right) \cup\left(S^{l-2} \times S^{\prime}\right) \times D^{2}$ where $\varphi^{\prime}: S^{2 l-1} \rightarrow S^{2 l-1}$ is a diffeomorphism covering $\varphi$ and $\bar{\varphi}^{\prime}(x, t)=\left(\varphi^{\prime}(x), t+1\right)$. Now $\pi_{l-1}(\partial Y)=\pi_{l-1}(\overline{\partial Y})=H_{l-1}(\overline{\partial Y})=0$. 
And $\pi_{l}(\partial Y)=\pi_{l}(\overline{\partial Y})=H_{l}(\overline{\partial Y})=Z+Z$. Since there is a normal map $Y$ $\rightarrow P_{l+1}$, it follows that $\overline{\partial Y}$ is $s$-parallelizable, and so that $\overline{\partial Y}$ is homotopy equivalent to $S^{l} \times S^{l}$. The lemma now follows.

Since $\partial Y_{3} \cong \partial Y$, Lemma 2.2 implies that $\partial Y_{3}$ is the quotient of a smooth free involution on a homotopy $S^{l} \times S^{l}$. Let $m$ be the mapping cylinder of $\alpha_{3} \mid \partial Y_{3}: \partial Y_{3}$ $\rightarrow P_{l}$. It follows that $\left(m, \partial Y_{3}\right)$ is a Poincaré duality pair. The map $\alpha_{3}: Y_{3} \rightarrow P_{l}$ allows us to extend id: $\partial Y_{3} \rightarrow \partial Y_{3}$ to $B:\left(Y_{3}, \partial Y_{3}\right) \rightarrow\left(m, \partial Y_{3}\right)$, a normal degree one map. Since the Wall group $L_{2 l+1}\left(Z_{2},+\right)$ is trivial, there is an interior cobordism from $B$ to $B^{\prime}:\left(Y_{4}, \partial Y_{4}\right) \rightarrow\left(m, \partial Y_{3}\right)$ with $\partial Y \cong \partial Y_{3} \cong \partial Y_{4}$ and $Y_{4} \rightarrow m$ a homotopy equivalence. We may embed $P_{l} \cong Y_{4}$ by Haefliger's embedding theorem. Then $\mathrm{Wh}\left(Z_{2}\right)=0$ implies that $Y_{4} \cong E\left(v\left(P_{l}: Y_{4}\right)\right) \cong E\left((l-1) y_{l}+2\right)$.

Let $Y^{\prime}$ be a smooth submanifold of int $Y$ such that $\operatorname{clos}\left(Y-Y^{\prime}\right) \cong \partial Y \times I$. Then $Y^{\prime} \cong Y$ so $\partial Y^{\prime} \cong \partial Y \cong \partial Y_{4}$. By means of a diffeomorphism $\partial Y^{\prime} \cong \partial Y_{4}$ paste $\operatorname{clos}\left(X-Y^{\prime}\right)$ and $Y_{4}$ to obtain a new manifold $X^{\prime}$ with $\partial X^{\prime} \cong \partial X$. We have the inclusion $P_{2 l-1} \subset T(\varphi)=\partial X \cong \partial X^{\prime} \subset X^{\prime}$.

Lemma 2.3. The inclusion $P_{2 l-1} \subset X^{\prime}$ is a homotopy equivalence.

Proof. $X^{\prime}$ is diffeomorphic to the union of $E\left(l y_{l-1} \times D^{2}\right)$ and $E\left(v\left(P_{l}: Y_{4}\right)\right)$ pasted by means of an embedding $S\left(l y_{l-1} \times D^{2}\right) \subset S\left(v\left(P_{l}: Y_{4}\right)\right)$. By the SeifertVan Kampen theorem immediately $P_{2 l-1} \subset X^{\prime}$ induces an isomorphism of fundamental groups. Let $P_{l}^{\prime}, P_{l-2} \subset P_{2 l-1}$ be disjoint standard copies of $P_{l}$ and $P_{l-2}$. Recall that $E\left((l+1) y_{l-2}\right)$ is a tubular neighborhood of $P_{l-2} \subset P_{2 l-1}$, and that $P_{l} \subset P_{2 l-1}-P_{l-2}$ is a homotopy equivalence, and that the composition $S^{l} \subset$ $S\left((l+1) y_{l-2}\right) \subset P_{2 l-1}-P_{l-2}$ is homotopy equivalent to the double cover $S^{l} \rightarrow P_{l}$. Using this fact and the Mayer-Vietoris sequence for the double cover $\bar{X}^{\prime}$ of $X^{\prime}$, it follows immediately that the inclusion $S^{2 l-1} \subset \bar{X}^{\prime}$ is a homotopy equivalence. The lemma follows.

By means of a boundary collar, we may embed $P_{2 l-1} \subset$ int $X^{\prime}$, isotopically to our original embedding $P_{2 l-1} \subset \partial X^{\prime} \subset X^{\prime}$. A tubular neighborhood of the new embedding is $P_{2 l-1} \times D^{2}$, and it follows from Lemma 2.3 that $\operatorname{clos}\left(X^{\prime}\right.$ $\left.-P_{2 l-1} \times D^{2}\right)$ is an $h$-cobordism. Moreover, it contains a trivial $h$-cobordism $P_{2 l-1} \times I$-given by the boundary collar above-from $P_{2 l-1} \times 0 \subset \partial X^{\prime}$ to $P_{2 l-1}$ $\times 1 \subset P_{2 l-1} \times S^{1}$. Let $P_{2 l-1} \times 0 \times[-1,1]$ be a tubular neighborhood of $P_{2 l-1} \times 0$ in $\partial X^{\prime}$. Then there is an embedding $P_{2 l-1} \times I \times[-1,1]$ which is a tubular neighborhood of $P_{2 l-1} \times I$ in $\operatorname{clos}\left(X^{\prime}-P_{2 l-1} \times D^{2}\right)$. Now $\left(\operatorname{clos}\left(X^{\prime}-P_{2 l-1} \times D^{2}\right.\right.$ $\left.\left.-P_{2 l-1} \times I \times[-1,1]\right), P_{2 l-1} \times I \times\{-1,1\}\right)$ is a relative $h$-cobordism with fundamental group $Z_{2}$. Since $\mathrm{Wh}\left(Z_{2}\right)=0$, the $s$-cobordism theorem states that the exhibited product structure on the boundary of the $h$-cobordism extends to all of $\operatorname{clos}\left(X^{\prime}-P_{2 l-1} \times D^{2}-P_{2 l-1} \times I \times[-1,1]\right)$. Thus $\operatorname{clos}\left(X^{\prime}-P_{2 l-1} \times D^{2}\right) \cong P_{2 l-1}$ $\times S^{1} \times I$ and $\partial X^{\prime} \cong P_{2 l-1} \times S^{1}$. And then $T(\varphi) \cong \partial X^{\prime} \cong P_{2 l-1} \times S^{1}$.

Lemma 2.4. Let $M$ be a smooth connected closed manifold with $\mathrm{Wh}\left(\pi_{1}(M)\right)=0$, and let $\varphi, \psi: M \rightarrow M$ be diffeomorphisms. Suppose that there is a diffeomorphism

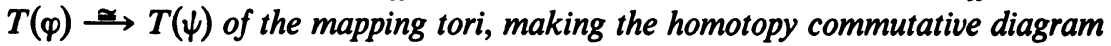




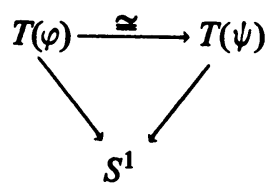

where the vertical maps are the natural fibrations. Then $\psi$ is concordant to a conjugate of $\varphi$.

Proof. Let $f: T(\varphi) \rightarrow T(\psi)$ be the diffeomorphism. Then there is a diffeomorphism $\bar{f}: M \times R \rightarrow M \times R$ making the following $\mathrm{d}^{\bar{\phi}}$,ram commute

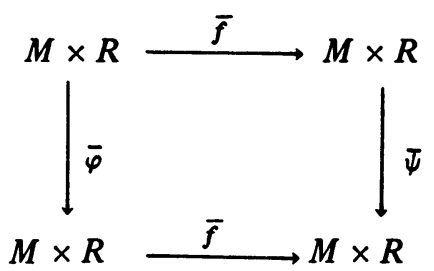

where $(\bar{\varphi})(x, t)=(\varphi(x), t+1)$ and similarly for $\zeta(\bar{\psi})$. By taking $i \in Z_{+}$large enough, we see that $M \times 0$ and $\bar{f}(M \times i)$ bound an $h$-bordism in $M \times R$. Since $\mathrm{Wh}\left(\pi_{1}(M)\right)=0$, that is an $s$-cobordism and there is an isotopy $h_{t}$ from $M \times i \stackrel{j}{\rightarrow} M \times R$ to $(x, i) \rightarrow\left(g_{0}(x), 0\right)$ where $g_{0}$ is some diffeomorphism. The isotopy may be extended to an isotopy $H_{t}$ of a bicollar of $M \times i$ in $M \times R$ to produce $G_{0}: M \times(i-\varepsilon, i+\varepsilon) \rightarrow M \times R$ with $G_{0}(x, t)=\left(g_{0}(x), t-i\right)$. The isotopy $\bar{\psi} \circ H_{i} \circ \bar{\varphi}^{-1}$ on $M \times(i+1-\varepsilon, i+1+\varepsilon)$ is an isotopy from $M \times(i+1$ $-\varepsilon, i+1+\varepsilon) \stackrel{J}{\rightarrow} M \times R$ to $G_{1}$ where $G_{1}(x, t)=\left(g_{1}(x), t-i\right)$. By means of the isotopy extension theorem, the isotopy $H_{t} \cup \bar{\psi} \circ H_{t} \circ \bar{\varphi}^{-1}$ may be extended to an isotopy $H_{t}^{\prime}: M \times(i-\varepsilon, i+1+\varepsilon) \rightarrow M \times R$ such that $\bar{\psi} \circ H_{t}^{\prime}=H_{t}^{\prime} \circ \bar{\phi}$ wherever both sides are defined. Then, since the translates of $M \times(i-\varepsilon, i+1+\varepsilon)$ by $\bar{\varphi}$ or $\bar{\psi}$ cover $M \times R, H_{t}^{\prime}$ has a unique extension to an isotopy $H_{t}^{\prime \prime}: M \times R$ $\rightarrow M \times R$, such that $\bar{\psi} \circ H^{\prime \prime}{ }_{t}=H^{\prime \prime}, \bar{\varphi}$, from $\bar{f}$ to a new diffeomorphism $\bar{g}$ : $M \times R \rightarrow M \times R$ making the diagram

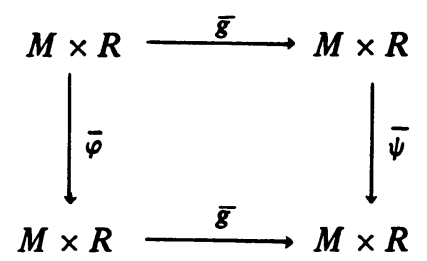

commute. Since the diffeomorphism $(x, t) \rightarrow(x, t-i)$ is also equivariant, we may assume that there is a diffeomorphism $\bar{g}: M \times R \rightarrow M \times R$ making the diagram above commute and satisfying $\bar{g}(x, 0)=\left(g_{0}(x), 0\right)$ and $\bar{g}(x, 1)=\left(g_{1}(x)\right.$, 1). We have in addition that $\bar{g}: M \times I \rightarrow M \times I$ so that $g_{0}$ and $g_{1}$ are concordant. Thus $\psi=g_{1} \circ \varphi \circ g_{0}^{-1}$, which is concordant to $g_{1} \circ \varphi \circ g_{1}^{-1}$, and the lemma is proved. 
To apply Lemma 2.4 to the diffeomorphism $T(\varphi) \cong P_{21-1} \times S^{1}=T$ (id), we must take into account the possibility that the diagram

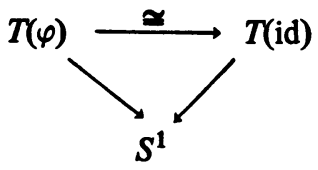

might not homotopy commute. Define $P O(2 l-1)$ to be the quotient of $S O(2 l-1)$ by the normal subgroup $\{\mathrm{id},-\mathrm{id}\}$. Let $\alpha: S^{1} \rightarrow P O(2 l-1)$ be a smooth loop that lifts to a path from id to $-\mathrm{id}$ in $P O(2 l-1)$. There is a smooth action

$$
P O(2 l-1) \times P_{2 l-1} \rightarrow P_{2 l-1} .
$$

Define $\bar{\alpha}: P_{2 l-1} \times S^{1} \rightarrow P_{2 l-1} \times S^{1}$ by $\bar{\alpha}(x, t)=(\alpha(t) \cdot x, t)$. Then $\bar{\alpha}$ is a diffeomorphism. Define another diffeomorphism $\bar{\beta}: P_{2 l-1} \times S^{1} \rightarrow P_{2 l-1} \times S^{1}$ by $\bar{\beta}(x, t)$ $=\left(x, t^{-1}\right)$. Then following the given diffeomorphism $T(\varphi) \rightarrow T$ (id) with $\bar{\alpha}$ or $\bar{\beta}$ or both or neither, we obtain a diffeomorphism $T(\varphi) \rightarrow T$ (id) making the diagram

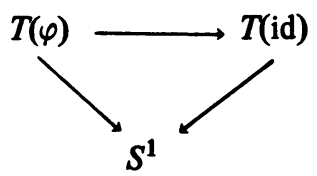

commute; then Lemma 2.4 implies that $\varphi$ is concordant to the identity, and the proposition is proved.

3. Epimorphism of $t$. So far we have obtained $t: \pi_{0}$ Diff: $\left(P_{2 l-1}, P_{-2}\right) \rightarrow \Omega_{2 l}(v)$, a monomorphism. It remains to be seen that $t\left(\pi_{0} \operatorname{Diff:}\left(P_{2 l-1}, P_{l-2}\right)\right)=\operatorname{Tors}\left(\Omega_{2 l}(v)\right)$; then Theorem 1 will be proved. To see that $t\left(\pi_{0} \operatorname{Diff}:\left(P_{2 l-1}, P_{-2}\right)\right) \subset \operatorname{Tors}\left(\Omega_{2 l}(v)\right)$ we simply notice that the index of $T(\varphi)$ is zero for any $\varphi \in \operatorname{Diff}\left(P_{2 l-1}\right)$ so that the image of $t$ is contained in the kernel of the Hirzebruch homomorphism $\Omega_{2 l}(v) \stackrel{\text { Index }}{\longrightarrow} Z$. Then we apply the following lemma.

Lemma 3.1. $\operatorname{Tors}\left(\Omega_{2 l}(v)\right)=\operatorname{kernel}\left(\Omega_{2 l}(v) \stackrel{\operatorname{Index}}{\longrightarrow} Z\right)$.

Proof. $H^{*}(P[l]: Q)=Q\left[P_{(l+2) / 4}, P_{(l+6) / 4}, \ldots\right]$ where $P_{i}$ is the pullback of the $i$ th Pontrjagin class of $B S O$. Thus $H_{2 l}(P[l]: Q)=Q$ and is detected by $P_{l / 2}$. Consequently $H_{2 l}(P[l] Q)$ is detected by the Hirzebruch index polynomial $L_{l / 2}$ with kernel

$$
\left(H_{2 l}\left(P[l] \stackrel{\iota^{l / 2}}{\longrightarrow} Q\right)=\text { Tors } H_{2 l}(P[l])\right) .
$$

Then by the Thom isomorphism theorem and the Hurewicz isomorphism $\pi_{*}^{s}(x) \otimes Q \stackrel{\approx}{\longrightarrow} H_{*}(X: Q)$ for any $X$, Tors $\left(\Omega_{2 l}(v)\right)$ is the kernel of Index $=L_{l / 2}$, and the lemma is proved.

Finally the following proposition completes the proof of Theorem 1. 
Proposition 2. $t: \pi_{0} \operatorname{Diff}\left(P_{2 l-1}, P_{l-2}\right) \rightarrow$ Tors $\Omega_{2 l}(v)$ is an epimorphism.

Proof. It follows as in [8] that an element $\mu$ in the kernel of Index: $\Omega_{2 l}(v) \rightarrow Z$ is represented by a normal map $Q(\zeta) \rightarrow P[l]$ where $\zeta: M(\zeta) \rightarrow M(\zeta)$ is a smooth free involution of a closed smooth $s$-parallelizable manifold $M(\zeta)$ homotopy equivalent to $S^{l} \times S^{l}$. To identify the map $Q(\zeta) \rightarrow P[l]$ recall from [8] that there is an embedding $P_{l} \subset Q(\zeta)$ which lifts to $S^{l} \subset M(\zeta)$ homotopy equivalent to the inclusion of a factor $S^{l} \subset S^{l} \times S^{l}$. Recall also, from [9], that there is an embedding $S^{l} \subset Q(\zeta)$ that meets $P_{l}$ transversally at one point. The union $P_{l} \vee S^{l} \subset Q(\zeta)$ is an $l$-skeleton of $Q(\zeta)$. The inclusion $P_{l} \subset P_{\infty}$ together with the map $S^{l} \rightarrow^{*} \in P_{\infty}$ defines a lift $P_{l} \vee S^{l} \rightarrow P_{\infty}$ of the oriented normal bundle map $Q(\zeta) \rightarrow B S O$ restricted to $P_{l} \vee S^{l}$. The composition $P_{l} \vee S^{l} \rightarrow P_{\infty} \rightarrow P[l]$ is a lift of the same restriction, and by obstruction theory there is a unique lift $Q(\zeta) \rightarrow P[l]$ of the oriented normal bundle map, extending $P_{l} \vee S^{l} \rightarrow P[l]$. This is the map $Q(\zeta) \rightarrow P[l]$. (There were four choices involved, given $Q(\zeta)$ alone: there are two orientations of $Q(\zeta)$ and there are two possible isotopy classes of embeddings $P_{l} \subset Q(\zeta)$ lifting to factor inclusions $S^{l} \subset S^{l} \times S^{l}$, but by making the choices suitably, one obtains a map $Q(\zeta) \rightarrow P[l]$ representing $\mu$.)

Define a free $Z_{2}$ action on $S^{2 l-1} \times D^{2}$ by letting the involution be $-1 \times 1$. Let $X=S^{2 l-1} \times D^{2} / Z_{2}$. Then there are disjoint copies of $P_{l}$ and $P_{2 l-1}$ in $\partial X$, and $v\left(P_{l}: \partial X\right)=(l-1) y_{l-1}+1=v\left(P_{l}: Q(\zeta)\right)$. Let $E_{1}$ be a tubular neighborhood of $P_{l}$ in $Q(\zeta)$ and $E_{2}$ a tubular neighborhood disjoint from $P_{2 l-1}$ of $P_{l}$ in $\partial X$. There is a diffeomorphism $h: E_{2} \stackrel{\cong}{\rightrightarrows} E_{1}$. We may assume that the normal map $Q(\zeta)$ $\rightarrow P(l)$ restricted to $E_{1}$ factors through a map $E_{1} \rightarrow P_{\infty}$. There is a normal map $X \rightarrow P_{\infty}$ which restricts to $E_{2} \rightarrow E_{1} \rightarrow P_{\infty}$. Thus we obtain a $v$-cobordism $Y=Q(\zeta) \times I \cup X / \sim \rightarrow P[l]$ where $(h(x), 1) \sim x$ for $x \in E_{2}$. Corners may be rounded on $Y$ so that $Y$ is a smooth $v$-cobordism. We have $\partial Y=Q(\zeta)+\partial_{1} Y$. As in [8], the double cover of $Q(\zeta)$ is $\left(S^{l} \times S^{l}\right) \# \Sigma$ where \# denotes connected sum and $\Sigma$ is a homotopy $2 l$-sphere. It is easy to see that then the corresponding double cover of $\partial_{1} Y$ is $\left(S^{1} \times S^{2 l-1}\right) \# \Sigma$. Then the universal cover of $\partial_{1} Y$ is $R \times S^{2 l-1}$. It is easy to check that the cover $\overline{\partial_{1} Y}$ of $\partial_{1} Y$ with fundamental group $Z_{2}$ is homotopy equivalent to $P_{2 l-1}$. But the embedding $P_{2 l-1} \subset \partial_{1} Y$ lifts to an embedding $P_{2 l-1} \subset \overline{\partial_{1} Y}$; since $\overline{\partial_{1} Y}$ is orientable, it follows that $\overline{\partial_{1} Y} \cong R \times P_{2 l-1}$. Let $\partial_{1} Y \stackrel{f}{\rightarrow} S^{1}$ be a map classifying the cover $\overline{\partial_{1} Y} \rightarrow \partial_{1} Y$. Notice that on a tubular neighborhood $I \times P_{2 l-1}$ of $P_{2 l-1}$ in $\partial_{1} Y$ that map may be taken to be $\mathrm{pr}_{1}: I \times P_{2 l-1} \rightarrow I \subset S^{1}$. Then $f^{-1}\left(\operatorname{clos}\left(S^{1}-I\right)\right)$ is an $h$-cobordism with ends $P_{2 l-1}$, and so a copy of $I \times P_{2 l-1}$. But then $\partial_{1} Y=T(\varphi)$ where $\varphi: P_{2 l-1} \rightarrow P_{2 l-1}$ is some diffeomorphism and the inclusion $P_{2 l-1} \subset \partial_{1} Y$ coming from $P_{2 l-1} \subset \partial X$ $-E_{2}$ is the standard inclusion $P_{2 l-1} \subset T(\varphi)$. Then the commutative diagram

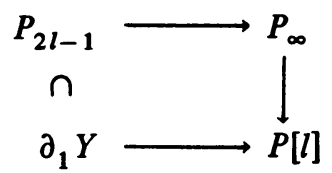


becomes the commutative diagram

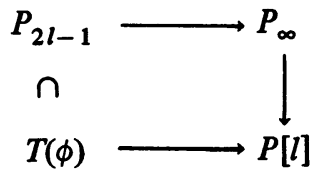

We may assume by Lemma 1.2 that $\varphi \mid P_{l-2}=$ id. Then the diagram above restricts to

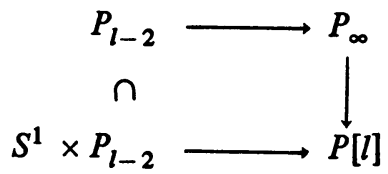

and we see that there is a unique lifting $S^{1} \times P_{l-2} \rightarrow P_{\infty}$ extending $P_{l-2} \rightarrow P_{\infty}$. Now, the embedding $S^{1} \subset S^{1} \times P_{l-2} \subset T(\varphi)=\partial_{1} Y$ was originally an embedding $S^{1} \subset D^{2} \subset X$. Since the map $T(\varphi) \rightarrow P[l]$ extends to $Y \rightarrow P[l]$, the map $S^{1} \rightarrow P[l]$ extends to $D^{2} \subset X \times P[l]$ so that the map $S^{1} \rightarrow P[l]$ is trivial. Then we may assume that $S^{1} \times P_{l-2} \rightarrow P_{\infty}$ is $S^{1} \times P_{l-2} \rightarrow P_{l-2} \rightarrow P_{\infty}$. Thus the normal map $T(\varphi) \rightarrow P[l]$ representing $N$ is precisely the map $v(\varphi)$ in the definition of $t(\varphi)$. Consequently $t(\varphi)=\mu$ and the proof of Proposition 2 is complete.

\section{BIBLIOGRAPHY}

1. P. Antonelli, D. Burghelea and P. J. Kahn, The concordance homotopy groups of geometric automorphism groups, Lecture Notes in Math., vol. 215, Springer-Verlag, Berlin and New York, 1970.

2. M. Kervaire and J. W. Milnor, Groups of homotopy spheres. I, Ann. of Math. (2) 77 (1963), 504-537. MR 26 \#5584.

3. R. K. Lashof, Poincaré duality and cobordism, Trans. Amer. Math. Soc. 109 (1963), pp. 257-277. MR 27 \#6281.

4. M. Mahowald, The metastable homotopy of $S^{n}$, Mem. Amer. Math. Soc. No. 72 (1967). MR 38 \#5216.

5. H. Schneider, Free involutions of homotopy $S^{[n / 2]} \times S^{[(n+1) / 2]}$ s, Thesis, University of Chicago, Chicago, Ill., 1972.

6. R. E. Stong, Notes on cobordism theory, Princeton Math. Notes, Princeton Univ. Press, Princeton, N.J., 1968. MR 40 \#2108.

7. C.T.C Wall, Surgery on compact manifolds, Academic Press, New York, 1970.

8. R. Wells, Free involutions of homotopy $S^{l} \times S^{l}$ s, Illinois J. Math. 15 (1971), 160-184. MR 42 \#6838.

9. - Some examples of free involutions of homotopy $S^{l} \times S^{b} s$, Illinois J. Math. 15 (1971), 542-550. MR 44 \#5980.

10.—, Cobordism groups of immersions, Topology 5 (1966), 281-294. MR 33 \#4946.

11.—_ Modification of intersections, Illinois J. Math. 11 (1967), 389-403. MR 36 \#896.

12. E. Thomas, Seminar on fibre spaces, Lecture Notes in Math., no. 13, Springer-Verlag, Berlin and New York, 1966. MR 34 \#3582.

13. J. P. May, The cohomology of the Steenrod algebra; stable homotopy groups of spheres, Bull. Amer. Math. Soc. 71 (1965), 377-380. MR 32 \#3060.

14. J. M. Cohen, Some results on the stable homotopy groups of spheres, Bull. Amer. Math. Soc. 72 (1966), 732-735. MR 35 \# 1022.

DEPARTMENT OF MATHEMATICS, PENNSYLVANIA STATE UNIVERSITY, UNIVERSITY PARK, PENNSYLVANIA 16802 